\title{
ERCP Scopes: What Can We Do to Prevent Infections?
}

\author{
William A. Rutala, PhD, MPH; ${ }^{1,2}$ David J. Weber, MD, MPH ${ }^{1,2}$
}

(See the article by Wendorf et al, on pages 634-642)

In the past year, multiple reports of outbreaks have led the Food and Drug Administration (FDA), the Centers for Disease Control and Prevention (CDC), and national news to raise awareness among the public and healthcare professionals that the complex design of duodenoscopes, used primarily for endoscopic retrograde cholangiopancreatography (ERCP), may impede effective reprocessing. ${ }^{1,2}$ Several recent publications, including the paper in this issue of ICHE by Wendorf et al, ${ }^{3}$ have associated multidrug-resistant (MDR) bacterial infections, especially carbapenemase-resistant Enterobacteriaceae (CRE), in patients who have undergone ERCP with reprocessed duodenoscopes. ${ }^{3-5}$ Unlike other endoscope-associated outbreaks, these recent outbreaks occurred even when the manufacturer's instructions and professional guidelines were followed correctly. ${ }^{3,4}$ The purpose of this commentary is 3-fold: (1) to briefly discuss the outbreak described by Wendorf et al; (2) to discuss what alternatives exist today that might improve the safety margin associated with duodenoscope reprocessing; and (3) to discuss how to prevent future outbreaks associated with ERCP endoscopes and other gastrointestinal (GI) endoscopes. ${ }^{6}$

In this issue of ICHE, Wendorf et al report on an outbreak involving a novel AmpC-producing E. coli strain that occurred among patients undergoing ERCP for severe biliary disease. Using pulsed-field gel electrophoresis, cultures obtained from 2 of 8 reprocessed ERCP scopes were found to harbor AmpC-producing E. coli that matched the patient isolates. The endoscope reprocessing procedures were reviewed and no lapses were identified. ${ }^{3}$

The elevator channel in duodenoscopes is unique to sideviewing endoscopes. It orients catheters, guide wires, and accessories into the endoscopic visual field. ${ }^{6}$ This separate channel is complex in design and has crevices that are difficult to access with a cleaning brush, which may impede effective reprocessing. ${ }^{2}$ Based on this characteristic and other recent studies, it is likely that MDR pathogens are acting as a "marker" or "indicator" organism for ineffective reprocessing of the complex design of duodenoscopes, which poses an infectious risk to patients. It is unclear whether echoendoscopes that have an elevator channel for the same reasons as ERCP scopes (directing accessories) pose the same disinfection challenges and similar infectious risk; these scopes are used to violate otherwise sterile spaces to obtain diagnostic samples and for therapeutic interventions.

The key questions raised by this study and others are these: Are current endoscope reprocessing guidelines adequate to ensure a GI endoscope devoid of potential pathogens? Is it impossible to ensure reliably high-level disinfection (HLD) of endoscopes with long, narrow channels, right-angle turns, and heavy microbial contamination $\left(10^{7}-10^{10}\right.$ bacteria) and components that are difficult to clean and disinfect (eg, elevator channels)? To examine these questions, we briefly review the current knowledge on endoscope reprocessing and then offer recommendations. First, endoscopes are semicritical items that require at least HLD. ${ }^{7,8}$ Because flexible GI endoscopes are currently heat labile, only HLD with chemical agents or lowtemperature sterilization technologies are possible. ${ }^{7}$ Unfortunately, at present, no solution exists that has been proven to eliminate the risk of microbial contamination associated with duodenoscopes. For example, no low-temperature sterilization technology achieves a sterility assurance level (SAL) of $10^{-6}$ for GI endoscopes such as duodenoscopes. Second, more healthcare-associated outbreaks have been linked to contaminated endoscopes than to any other reusable medical device. ${ }^{7,9}$ However, until recently, these outbreaks have been traced to deficient practices such as inadequate cleaning and inappropriate disinfection (eg, failure to perfuse all channels), to damaged endoscopes or flaws in the design of endoscopes (eg, duodenoscope elevator channel), and to automated endoscope reprocessors (AERs). ${ }^{7,9}$ Reprocessing failures have led to patient notifications and bloodborne pathogen testing in dozens of instances. ${ }^{10}$ Third, evidence-based endoscope reprocessing guidelines have been prepared by professional organizations, and the CDC as well as past data suggest that rigorous adherence to these guidelines would result in a pathogen-free endoscope. ${ }^{7,8}$ Unfortunately, further data demonstrate that all of the steps associated with manual endoscope reprocessing are rarely performed and that some

Affiliations: 1. Hospital Epidemiology, University of North Carolina Health Care, Chapel Hill, North Carolina; 2. Division of Infectious Diseases, UNC School of Medicine, Chapel Hill, North Carolina.

Received April 1, 2015; accepted April 1, 2015

(c) 2015 by The Society for Healthcare Epidemiology of America. All rights reserved. 0899-823X/2015/3606-0005. DOI: 10.1017/ice.2015.98 
essential steps (eg, brushing all endoscope channels and components) are not commonly performed. ${ }^{11}$ Endoscope reprocessing was improved with the use of AERs as most steps were automated. ${ }^{7}$ Fourth, endemic transmission of infections associated with GI endoscopes may go unrecognized due to inadequate surveillance of outpatient procedures, the long lag time between colonization and infection, and a low frequency of infection. Additionally, the risk for some procedures might be lower than others (eg, colonoscopy versus ERCP where normally sterile areas are contaminated in the latter). In the outbreak reported by Wendorf et al, the presence of an unusual pathogen (AmpC-producing E. coli) resulted in an investigation and recognition that duodenoscopes were the source of the outbreak. ${ }^{3}$

Importantly, the margin of safety associated with reprocessing endoscopes is minimal or nonexistent. GI scopes are heavily contaminated with microbes. Studies have shown that the internal channel of GI endoscopes, including duodenoscopes, may contain $10^{7-10}\left(7-10 \log _{10}\right)$ enteric microorganisms. ${ }^{12,13}$ Investigations have demonstrated that the cleaning step in endoscope reprocessing results in a $2-6 \log _{10}$ reduction of microbes and that the HLD step results in another 4-6 $\log _{10}$ reduction of mycobacteria for a total 6-12 $\log _{10}$ reduction of microbes. ${ }^{12-14}$ Thus, the margin of safety associated with cleaning and HLD of GI endoscopes is minimal or nonexistent (level of contamination: $4 \log _{10}$ [maximum contamination, minimal cleaning/HLD] to $-5 \log _{10}$ [minimum contamination, maximum cleaning/HLD]). Therefore, any deviation from proper reprocessing (eg, in crevices associated with the elevator channel) could lead to failure to eliminate contamination with a possibility of subsequent patient-to-patient transmission. This low (or nonexistent) margin of safety associated with endoscope reprocessing compares to the $17 \log _{10}$ margin of safety associated with cleaning and sterilizing surgical instruments.

What should we do now? Unfortunately, there is currently no single, simple, and proven technology or prevention strategy that hospitals can use to guarantee patient safety. Of course, we must continue to emphasize the enforcement of evidenced-based practices, including equipment maintenance and routine audits with at least yearly competency testing of reprocessing staff. ${ }^{7,8}$ All reprocessing personnel must be knowledgeable and thoroughly trained on the reprocessing instructions for duodenoscopes. This training includes the new recommendations to use a new small bristle cleaning brush and for additional flushing and cleaning steps of the elevator channel. ${ }^{15}$ Although these steps were described as "validated," no data were presented on the ability of these new cleaning recommendations to yield an ERCP scope devoid of bacteria. We must do more or additional outbreaks will continue. We must obtain additional information on the frequency and level of microbial contamination of endoscopes that have been cleaned and disinfected using HLD and strict adherence to current guidelines. If endoscopes are found to be contaminated with potential pathogens (eg, enteric Gramnegative bacilli), the clinical impact of such contamination needs to be quantified. In addition, based on the studies by Wendorf et al and others, ${ }^{3-5}$ it would be reasonable to consider periodic microbiologic surveillance of duodenoscopes to assess microbial contamination as one component of a prevention strategy. However, culture results are delayed 2-3 days, and many questions related to microbiologic surveillance remain: What cutoff should be used to define proper disinfection (eg, 0 pathogens or a higher number $[\mathrm{eg},<10 \mathrm{CFU}]$ of enteric pathogens per channel)? Should there be a separate cutoff based on relatively nonvirulent pathogens such as coagulase-negative staphylococci? What sampling scheme should be used to evaluate GI endoscopes (eg, all scopes or a sample of endoscopes)? If a hospital cultures 2 endoscopes of 10 and 1 endoscope is positive, do they reprocess all 10 endoscopes as 50\% of the sampled endoscopes are positive? If a hospital does periodic microbiologic culturing and $20 \%$ of sampled endoscopes are positive, what actions should an endoscopy unit undertake (eg, patient notification with an offer of bloodborne pathogen testing, stool examination for CRE, ethylene oxide [ETO] sterilization of positive endoscopes, and/or HLD followed by ETO sterilization of all duodenoscopes)? Has the staff been trained on culturing the duodenoscope channels as well as the elevator channel? Finally, is the trigger for further action based on the level of contamination or the frequency of contamination (ie, percent of endoscopes contaminated)? ${ }^{6}$ In addition, if a hospital decides to culture all endoscopes and quarantine endoscopes for 48-72 hours while awaiting culture results before using the scope, it must be recognized that the sensitivity of culturing the elevator channel of the scope or the scope is unknown (ie, how many microbes must contaminate the endoscope to yield a positive culture?).

Real-time monitoring methods need to be developed and validated to assess the effectiveness of cleaning and HLD as well as the risk of infection. Adenosine triphosphate (ATP) detection of effluent has been proposed as a monitoring tool $^{16,17}$ for assessing cleaning because it detects organic residuals. However, ATP is not a good indicator of microbial contamination and has not been validated as a method to assess the risk for patient-to-patient transmission. A validation study of ATP used to audit cleaning of flexible endoscope channels used a $<200$ RLU benchmark for clean, which equates to $<4 \log _{10} \mathrm{CFUs} / \mathrm{cm}^{2}$ (or $10^{4} \mathrm{CFUs}$ ) $\left./ \mathrm{cm}^{2}\right)^{16}$ or $\sim 10^{6}$ CFUs per endoscope (ie, the surface area of an endoscope channel exceeds $100 \mathrm{~cm}^{2}$ ). Thus, an endoscope assessed as clean using ATP could still have a significant microbial load (eg, $10^{6}$ ). Third, endoscope manufacturers need to redesign their endoscopes (eg, elevator channel) to make them easier to achieve HLD or sterilization.

We predict that we will continue to see outbreaks associated with ERCP endoscopes and GI endoscopes if we incorporate only the enhanced strategies described above. One long-term solution to this infection prevention challenge would be to develop new endoscope reprocessing technologies that reliably result in sterilization of duodenoscopes and other GI 
TAвLE 1. Summary of Advantages and Disadvantages of High-Level Disinfection (HLD) and Sterilization Enhancements for Reprocessing Duodenoscopes

\begin{tabular}{|c|c|}
\hline Method & Advantages \\
\hline Steam; sterilization & $\begin{array}{l}\text { - Rapidly microbicidal } \\
\text { - Least affected by organic/inorganic soils } \\
\text { - Rapong sterilization processes listed } \\
\text { - Racle time }\end{array}$ \\
\hline $\begin{array}{c}\text { Hydrogen peroxide gas } \\
\text { plasma; sterilization }\end{array}$ & $\begin{array}{l}\text { - Cycle time is } \geq 28 \text { minutes and no } \\
\text { aeration necessary } \\
\text { - Used for heat- and moisture-sensitive } \\
\text { items since process temperature }<50^{\circ} \mathrm{C} \\
\text { - Compatible with most medical devices }\end{array}$ \\
\hline
\end{tabular}

$100 \%$ ETO; sterilization after HLD, microbiologic surveillance

Vaporized hydrogen peroxide; sterilization

HLD only (using FDA-cleared HLD such as OPA and glut)

Double HLD (back-to-back), microbiologic surveillance

Liquid chemical sterilant processing system using peracetic acid, rinsed with extensively treated potable water, microbiologic surveillance

HLD, microbiologic surveillance
- Single-dose cartridge and negativepressure chamber minimizes the potential for gas leak and ETO exposure

- Simple to operate and monitor

- Compatible with most medical materials

- Major endoscope manufacturer offers ETO as sterilization option

- Ideally, should be used after standard HLD

- Some data demonstrate reduced infection risk with HLD followed by ETO

- Fast cycle time, $55 \mathrm{~min}$

- Used for heat and moisture sensitive items (metal and nonmetal devices)

- HLD inactivates MDR organisms including CREs

- Current standard of care

- Wide availability

- HLD inactivates MDR organisms including CREs

- Wide availability

- A second HLD cycle may reduce or eliminate microbial contaminants remaining from first cycle

- HLD/chemical sterilant inactivate MDR organisms including CREs

- Offered as liquid chemical sterilant processing option

- HLD inactivates MDR organisms including CREs

- Microbiologic surveillance offered as supplement by CDC
Disadvantages

- Deleterious for heat-sensitive instruments

- At present, cannot be used as current GI scopes are not heat resistant

- Endoscope or medical device restrictions based on lumen internal diameter and length

- GI scopes cannot be processed

- No microbicidal efficacy data proving SAL $10^{-6}$ achieved

- Studies question microbicidal activity in presence of organic matter/salt

- May damage endoscope

- Requires aeration time to remove ETO residue

- Only $20 \%$ of US hospitals have ETO on site

- Lengthy cycle/aeration time

- No microbicidal efficacy data proving SAL $10^{-6}$ achieved

- Studies question microbicidal activity in presence of organic matter/salt

- ETO is toxic, a carcinogen, flammable

- May damage endoscope

- Endoscope or medical device restrictions based on lumen internal diameter and length

- GI scopes cannot be processed

- No microbicidal efficacy data proving SAL $10^{-6}$ achieved

- No data demonstrating microbicidal activity in presence of organic matter/salt

- May damage endoscope

- Based on recent ERCP outbreaks, infection risk related to device complexity and microbial load

- No enhancement to reduce infection risk associated with ERCP scopes

- Some HLD (eg, aldehydes) may cross-link proteins

- Based on recent ERCP outbreaks, infection risk related to device complexity and microbial load

- Some HLD (eg, aldehydes) may cross-link proteins

- Based on recent ERCP outbreaks, infection risk related to device complexity and microbial load

- Not considered sterile as not a terminal sterilization process and scope rinsed with extensively treated water

- Unclear whether peracetic acid will penetrate crevices in elevator channel and inactivate pathogens

- Based on recent ERCP outbreaks, infection risk related to device complexity and microbial load

- No data demonstrating reduced infection risk

- Sensitivity of microbiologic surveillance unknown

- 48-72 hours before culture results known

- No consensus regarding sampling scheme, $100 \%$ or $10 \%$ of scopes per week/per month

- No cutoff to define effective disinfection (0 GNR?) 
Table 1. Continued

\begin{tabular}{lll}
\hline Method & Advantages & Disadvantages \\
\hline HLD, ATP & - HLD inactivates MDR organisms & - Based on recent ERCP outbreaks, infection risk related \\
& including CREs & to device complexity and microbial load \\
& - Real-time monitoring tool & No data demonstrating reduced infection risk \\
& - Detects organic residue & - Does not detect microbial contamination \\
& & ATP not validated as risk factor for patient-to-patient \\
& & transmission \\
& & Unknown cutoff level to assure safety \\
\hline
\end{tabular}

NOTE. GI, gastrointestinal; ETO, ethylene oxide; SAL, sterility assurance level; FDA, Food and Drug Administration; OPA, ortho-phthalaldehyde; ERCP, endoscopic retrograde cholangiopancreatography; CRE, carbapenemase-resistant Enterobacteriaceae; CDC, Centers for Disease Control and Prevention; MDR, multidrug-resistant organism; GNR, Gram-negative rod; HLD, high-level disinfection or high-level disinfectant; ATP, adenosine triphosphate.

endoscopes via an FDA-cleared sterilization process that achieves a sterility assurance level (SAL) of $10^{-6}$. Some sterilization technologies that should be evaluated include ozone plus hydrogen peroxide vapor, nitrogen dioxide, ${ }^{18}$ supercritical $\mathrm{CO}_{2}$, peracetic acid vapor, gaseous chlorine dioxide, hydrogen peroxide gas plasma, and steam sterilization for heat-resistant endoscopes. These new technologies could greatly improve the margin of safety and eliminate patient risk. ETO, which was used by Epstein et $\mathrm{al}^{4}$ to terminate their outbreak, may represent a short-term solution for some hospitals, but it is not a satisfactory solution in the long term. Many hospitals no longer have ETO, the sterilization/aeration time is long (eg, 12-15 hours) and the process may eventually damage the endoscopes. Additionally, no studies in the peer-reviewed literature have demonstrated that we can depend on ETO (or other LTSTs) to sterilize a duodenoscope following only the cleaning portion of reprocessing instructions (ie, brushing and flushing). In fact, Alfa et $\mathrm{al}^{19}$ found the sterilization efficacies with $100 \%$ ETO or hydrogen peroxide gas plasma to be only $39.7 \%$ and $35 \%$, respectively, when serum and salt load were combined with a lumen carrier as the test challenge. When penicylinders were inoculated with 7 organisms in the presence of salt and serum, sterilization efficacies with $100 \%$ ETO and hydrogen peroxide gas plasma were $60.3 \%$ and $37 \%$, respectively. ${ }^{19,20}$ For this reason, if hospitals wish to consider ETO as an enhancement to their current ERCP reprocessing, each scope should be cleaned and high-level disinfected and dried per reprocessing instruction prior to ETO. Prior cleaning and HLD reduces the microbial load and organic challenge that may interfere with the effectiveness of ETO sterilization. It is unlikely, based on existing data, that ETO (or other LTSTs) will kill high numbers of pathogens in the presence of salt and serum in a lumened device.

Table 1 provides the advantages and disadvantages for various enhancements involving HLD or sterilization and Table 2 offers current and future alternatives. Unfortunately, many of these strategies may increase capital equipment and reprocessing costs, may cause changes in workflow and processes, and may cause a short-term shortage of duodenoscopes used in ERCP procedures. For these reasons and others (including medical-legal), we recommend that the infection prevention clinician seek executive-level support for the duodenoscope reprocessing enhancements needed to minimize the infection risk. Alternatively, development of sterile disposable GI endoscopes or a shift to other sterile diagnostic modalities (eg, capsule endoscopy, blood tests to detect GI cancer, etc.) would avoid outbreaks associated with HLD of endoscopes.

Improved prevention strategies must be urgently pursued. Despite the very low risk of MDR infection following ERCP, any avoidable infection risk must be eliminated. Manufacturers of endoscopes, AERs, high-level disinfectants, and low-temperature sterilization technologies and federal authorities (eg, CDC, FDA, National Institutes of Health) must be engaged by providing adequate resources to design and complete the necessary studies for determining the risks posed by current reprocessing of endoscopes and for developing new reprocessing methods/ practices. Infection prevention clinicians should be encouraged to report and publish additional outbreaks related to endoscopy, especially if current reprocessing methods are followed, so we can determine whether recent reports represent a larger problem or an anomaly. Thus, infection prevention associated with ERCP and GI scopes is multifaceted, and no single, immediately available strategy will eliminate this problem. However, the immediate risks can be minimized by a multicomponent strategy (eg, compliance with endoscope reprocessing guideline, HLD followed by ETO, and periodic microbiologic sampling). Only when we implement new technologies, such as equipment redesign, single-use sterile scopes, and sterilization of GI scopes with technology that achieves an SAL of $10^{-6}$, will we eliminate the risk of infection associated with duodenoscopes and other GI scopes.

Until these issues can be resolved, we should continue to provide GI endoscopic (eg, ERCP) procedures, which are an important diagnostic and therapeutic modality. These procedures should be performed while strictly adhering to current endoscope reprocessing guidelines ${ }^{3,4}$ with the enhancements offered (Table 1 and 2), and patients should be informed of the benefits and risks. 
t A ble 2. Challenges in High-Level Disinfection (HLD) of Gastrointestinal (GI) Endoscopes and Preventive Methods to Assure Safety

1. Challenges in GI Endoscope Design for HLD

- Heat labile (ie, require low temperature disinfection)

- Heavily contaminated with use

- Complex

- Long, narrow lumens

- Sharp angles (eg, right-angle bends)

- Occluded dead-end channels

- Difficult to clean and disinfect accessories (eg, elevator channel)

- Mated surfaces

- Springs and valves

- Rough or pitted surfaces

- Rapid turnover desired

- Damaged channels that impede microbial exposure to high-level disinfectant

2. Steps in HLD (rigorously adhere to current standards for cleaning and disinfection)

- Preclean at point of use

- Clean thoroughly

- HLD following manufacturer's recommendations

- Rinse thoroughly

- Dry completely

- Store properly

- Also assure competency of reprocessing staff (initial and at least annual training assessment)

3. Possible enhanced methods for reprocessing duodenoscopes currently available (priority ranked)

- Ethylene oxide sterilization after HLD with periodic microbiologic surveillance

- Double HLD with periodic microbiologic surveillance

- HLD with scope quarantine until negative culture

- Liquid chemical sterilant processing system using peracetic acid (rinsed with extensively treated potable water) with periodic microbiologic surveillance

- Other FDA-cleared low-temperature sterilization technology (provided material compatibility and sterilization validation testing performed using the sterilizer and endoscope) after HLD, with periodic microbiologic surveillance

- HLD with periodic microbiologic surveillance

4. Potential future methods to prevent GI endoscope-related outbreaks

- Steam sterilizable GI endoscopes

- New low-temperature sterilization methods proving SAL $10^{-6}$ achieved

- Disposable sterile GI endoscopes

- Improved GI endoscope design (to reduce or eliminate challenges listed in \#1 above)

- Use of nonendoscopic methods to diagnosis or treat disease (eg, capsule endoscopy, blood tests to detect GI cancer, stool DNA test)

\section{ACKNOWLEDGMENTS}

We thank Todd Huntley Baron, MD, Emily E. Sickbert-Bennett, PhD, and Ms. Judie Bringhurst, MSN (University of North Carolina Hospitals) for their helpful comments.

Financial support: No financial support was provided relevant to this article. Potential conflicts of interest: Dr. Rutala is a consultant for Clorox and Advanced Sterilization Products and has received an honorarium from 3M.

Dr. Weber is a consultant for Clorox.

Address correspondence to William A. Rutala, PhD, MPH, Director, Hospital Epidemiology, Occupational Health, and Safety Program, Room 1001 West Wing, UNC Health Care Chapel Hill, NC 27514 (brutala@unch.unc.edu).

\section{REFERENCES}

1. Interim protocol for healthcare facilities regarding surveillance for bacterial contamination of duodenoscopes after reprocessing. Centers for Disease Control and Prevention website. http://www. cdc.gov/hai/organisms/cre/cre-duodenoscope-surveillance-protocol. html. Published 2015. Accessed March 27, 2015.

2. Design of endoscopic retrograde cholangiopancreatography (ERCP) duodenoscopes may impede effective cleaning: FDA safety communication. Food and Drug Administration website.
http://www.fda.gov/MedicalDevices/Safety/AlertsandNotices/ucm 434871.htm. Published 2015. Accessed March 26, 2015.

3. Wendorf KA, Kay M, Baliga C, et al. Endoscopic retrograde cholangiopancreatography-associated AmpC Escherichia coli outbreak. Infect Control Hosp Epidemiol 2015;36:634-642.

4. Epstein L, Hunter JC, Arwady MA, et al. New Delhi metallo- $\beta$ lactamase-producing carbapenem-resistant Escherichia coli associated with exposure to duodenoscopes. JAMA 2014;312:1447-1455.

5. Carbonne A, Thiolet JM, Fournier S, et al. Control of a multihospital outbreak of KPC-producing Klebsiella pneumoniae type 2 in France, September to October 2009. Euro Surveill 2010:15: pii $=19734$.

6. Rutala WA, Weber DJ. Gastrointestinal endoscopes: a need to shift from disinfection to sterilization. JAMA 2014;312:1405-1406.

7. Rutala WA, Weber DJ. Healthcare Infection Control Practices Advisory Committee. Guideline for disinfection and sterilization in healthcare facilities, 2008. Centers for Disease Control and Prevention website. http://www.cdc.gov/ncidod/dhqp/pdf/guide lines/Disinfection_Nov_2008.pdf. Published 2008. Accessed March 27, 2015.

8. Petersen BT, Chennat J, Cohen J, et al. Multisociety guideline on reprocessing flexible GI endoscopes: 2011. Infect Control Hosp Epidemiol 2011;32:527-537. 
9. Kovaleva J, Peters FTM, van der Mei HC, Degener JE. Transmission of infection by flexible gastrointestinal endoscopy and bronchoscopy. Clin Reviews Microbiol 2013;26:231-253.

10. Rutala WA, Weber DJ. How to assess risk of disease transmission when there is a failure to follow recommended disinfection and sterilization principles. Infect Control Hosp Epidemiol 2007;28: 519-524.

11. Ofstead CL, Wetzler HP, Snyder AK, Horton RA. Endoscope reprocessing methods: a prospective study on the impact of human factors and automation. Gastroenterol Nurs 2010;33:304-311.

12. Roberts CG. Studies on the bioburden on medical devices and the importance of cleaning. In: Rutala WA, ed. Disinfection, sterilization and antisepsis: principles and practices in healthcare facilities. Washington, DC: Association for Professionals in Infection Control and Epidemiology; 2000:63-69.

13. Alfa MJ, Degagne P, Olson N. Worst-case soiling levels for patient-used flexible endoscopes before and after cleaning. Am J Infect Control 1999;27:392-410.

14. Rutala WA, Weber DJ. FDA labeling requirements for disinfection of endoscopes: a counterpoint. Infection Control Hosp Epidemiol 1995;16:231-235.
15. New reprocessing instructions for the Olympus TJF Q180V duodenoscope. Olympus America website. http://medical. olympusamerica.com/sites/default/files/pdf/150326_TJF-Q180V_ Customer_letter.pdf). Published 2015. Accessed March 27, 2015.

16. Alfa MJ, Fatima I, Olson N. Validation of adenosine triphosphate to audit manual cleaning of flexible endoscope channels. Am J Infect Control 2013;41:245-248.

17. American Society of Gastrointestinal Endoscopists Technology Committee. Technologies for monitoring the quality of endoscope reprocessing. Gastrointest Endosc 2014;80:369-372.

18. Schneider PM. New technologies and trends in sterilization and disinfection. Am J Infect Control 2013;41:S81-S86.

19. Alfa MJ, DeGagne P, Olson N, Puchalski T. Comparison of ion plasma, vaporized hydrogen peroxide, and $100 \%$ ethylene oxide sterilizers to the $12 / 88$ ethylene oxide gas sterilizer. Infection Control Hosp Epidemiol 1996;17:92-100.

20. Alfa MJ, Olson N, DeGagne P, Hizon R. New low temperature sterilization technologies: microbicidal activity and clinical efficacy. In: Rutala WA, ed. Disinfection, sterilization and antisepsis in health care. Washington, DC: APIC and Champlain, NY: Polyscience Publications; 1998:67-78. 